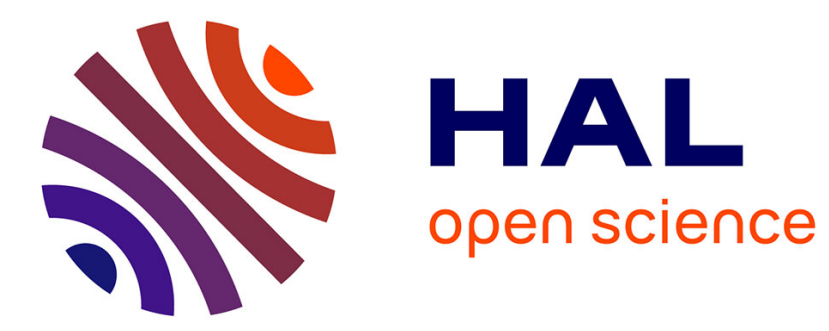

\title{
Radiographie et contextualisation des populismes
}

Nicolas Lebourg

\section{To cite this version:}

Nicolas Lebourg. Radiographie et contextualisation des populismes. La revue internationale et stratégique, 2017, 106 (2), pp.73-80. 10.3917/ris.106.0073 . hal-01696042

\section{HAL Id: hal-01696042 \\ https://hal.science/hal-01696042}

Submitted on 14 Feb 2018

HAL is a multi-disciplinary open access archive for the deposit and dissemination of scientific research documents, whether they are published or not. The documents may come from teaching and research institutions in France or abroad, or from public or private research centers.
L'archive ouverte pluridisciplinaire HAL, est destinée au dépôt et à la diffusion de documents scientifiques de niveau recherche, publiés ou non, émanant des établissements d'enseignement et de recherche français ou étrangers, des laboratoires publics ou privés. 
Nicolas Lebourg, « Radiographie et contextualisation des populismes », Olivier de France et Marc Verzeroli dir., «L'ordre international face aux contestations démocratiques », La Revue internationale et stratégique, $\mathrm{n}^{\circ} 106,2017, \mathrm{pp} .73-80$.

Le « Brexit » et l'élection de Donald Trump ont popularisé l'idée d'une « vague populiste » déferlant sur les démocraties. La campagne présidentielle française pourrait en être considérée comme un palier, puisque les 11 candidats se sont tous présentés comme voulant faire litière du personnel et du système politiques des trois dernières décennies - mais n'ont pas tous, pour autant, défini le peuple telle une unité homogène. Alors qu'il était commun, dans le débat français, de catégoriser comme populistes les opposants au « laissez-aller, laissez-faire », le candidat eurolibéral - Emmanuel Macron - comme l'ordolibéral - François Fillon - ont eux aussi adopté ce fameux « style » par lequel on définit souvent le populisme.

Pourtant, dans le même temps, l'action du président Trump butait à plusieurs reprises sur l'obstacle des contre-pouvoirs, soulignant la difficulté à exercer une gouvernance personnelle populiste au sein d'un régime libéral. En outre, le « Brexit » et la présidentielle française ne sauraient être aisément assimilés à une métamorphose populiste : fonctionnant sur le principe $50 \%$ plus une voix, ces processus démocratiques impliquent, par définition, la construction d'un bloc électoral interclassiste. Il ne s'agit donc pas de partir du principe d'une mue populiste de la vie politique occidentale pour comprendre le phénomène à l'œuvre. Mieux vaut privilégier l'observation contextualisée de la dynamique des mouvements populistes pour saisir ce qui se joue, tant l'objet « populisme » résonne différemment selon les histoires nationales. Pour mettre à jour ce quesignifie structurellement la perception d'une « droitisation de l'Occident », il importe d'observer les populismes en différenciant trois types de pays : ceux peu ou prou d'expérience libérale, ceux qui ont connu une dictature nationaliste et ceux qui en eurent une communiste.

\section{Les populismes en contextes libéraux}

Le mot « populisme » apparaît au XIXe siècle dans des sociétés amplement externes au clivage droite-gauche : la Russie et les États-Unis. Mais il connaît son heure de gloire après la Seconde Guerre mondiale avec les régimes nationalistes arabes et, surtout, sud-américains. Est ici présente une alchimie particulière : culte du chef charismatique mais absence de totalitarisme, réunion des couches sociales dans une hiérarchie voulue comme légitime, affirmation d'un État non soumis aux oligarchies, nationalisme non impérialiste, etc. Ces régimes ont souvent été taxés de «fascistes » dans le débat polémique, mais à l'évidence ces critères les excluent rationnellement de cette catégorie. Or, dans le même temps, les premiers mouvements populistes européens émergeaient 
depuis les décombres des fascismes (Autriche, Flandres, etc.), cette matrice radicale les ayant depuis menés vers un populisme identitaire.

On voit alors se forger l'étiquette de « national-populiste » pour décrire la réalité latino-américaine. Le premier, sans doute, à en faire usage en France est François Duprat, alors numéro deux du Front national (FN), qui, pour reprendre l'autodéfinition du parti que donnait Jean-Marie Le Pen, louait cette « droite nationale, sociale et populaire » latino-américaine ${ }^{1}$. D’ailleurs, l'apparition électorale des populismes se fait avant tout dans les années 1970 dans les pays nordiques (Danemark, Norvège), en réaction à l'État-providence. Ces populismes à matrice anti-fiscaliste ont évolué vers un identitarisme de la prospérité pouvant aller jusqu'à susciter des antagonismes intereuropéens au Luxembourg, le Mouvement national exigeait le départ des Français, tandis qu'en Suisse, l'Union démocratique du centre (UDC) et le Mouvement citoyens genevois (MCG) visent également les travailleurs frontaliers français. Enfin, les derniers populismes apparus ont une matrice agrarienne (Suisse, Finlande, etc.) qui a évolué, à partir des succès connus aux Pays-Bas par la liste Pim Fortuyn en 2002, vers un « hédonisme sécuritaire »- selon la formule de Gaël Brustier et Jean-Philippe Huelin ${ }^{2}$ - exigeant d'allier individualisme libéral et contexte sécuritaire. C'est alors l'émergence du néo-populisme, qui se différencie du national-populisme tel qu'apparu au XIXe siècle par son intégration des valeurs libérales à son altérophobie et à son projet organiciste deux traits quidemeurent dans le néo-populisme et qui confirment que ces mouvements ne sont pas exogènes au champ des extrêmes droites. La critique des musulmans ne se fait pas ici sur le thème du conservatisme chrétien, mais de la défense des libertés acquises par les femmes, par les gays, par les juifs. Le passage du national-populisme au néo-populisme marque ce vrai-faux paradoxe : l'extension des valeurs libérales au sein du champ extrême droitier, le passage de celui-ci de la critique du welfare state à celle du multiculturalisme.

Le FN français réussit à combiner les données de ces trois tendances, ce qui explique sans doute en bonne part la particularité de son succès. Si cette offre politique connaît une réception à un niveau jusque-là inégalé en France, c'est aussi qu'elle correspond à l'opinion, de par : l'hégémonie culturelle de la demande autoritaire - entre les attentats de janvier et de novembre $2015,88 \%$ des sondés estiment que l'« on a besoin d'un vrai chef en France pour rétablir l'ordre » et $40 \%$ accepteraient un « pouvoir politique autoritaire, quitte à alléger les mécanismes de contrôle démocratique s'exerçant sur le gouvernement $»^{3}$; la conversion de l'électorat aux conceptions populistes - une enquête d'octobre 2016 sur 17047 sondés proposant six items pour construire un score de populisme allant de 1 à 5 aboutit à une moyenne de 3,9 et enregistre les plus hauts scores 
parmi les personnes s'auto-positionnant aux extrêmes mais également au centre ${ }^{4}$, ce qui contribue à éclairer la stratégie d'Emmanuel Macron ; la dislocation, depuis 2008, du bloc social soutenant les politiques eurolibérales - avec une croissance de la précarité qui induit, chez les intéressés, un basculement électoral vers le populisme ${ }^{5}$, et le sentiment d'une correspondance entre la représentation d'un déclin national et d'un risque de déclassement personnel.

L'adaptation du phénomène populiste à une société nationale s'avère donc complexe, avec peu de règles transposables d'un pays à l'autre, ainsi qu'une difficulté à stabiliser l'offre populiste dans un certain nombre de territoires. Ce double problème est particulièrement visible si l'on s'attache à l'observation des faits populistes dans les anciennes dictatures d'extrême droite.

\section{Les populismes après les dictatures nationalistes}

Le seul pays européen ayant connu l'instauration d'une dictature d'extrême droite après 1945, la Grèce, est également le seul à voir le succès d'une formation d'extrême droite radicale. Fondée en 1980, Aube dorée ne reprend pourtant pas les codes de l'époque des colonels - un régime justement qualifié de populiste en son temps par la presse occidentale, mais en lien avec les néofascistes européens. Aube dorée a évolué du néo-paganisme à la défense de l'identité orthodoxe, mais promeut toujours un ethno-nationalisme impérialiste et représente un cas avéré de parti-milice. Son évolution récente, de 0,46\% des voix en 2009 à 9,32\% en 2014, est nettement corrélée à l'humiliation du pays consécutive à la crise financière. Le parti a profité de la normalisation du Rassemblement orthodoxe populaire - sécuritaire, anti-globalisation, xénophobe et islamophobe -, mais qui a perdu son attractivité après sa participation en 2011 à une coalition gouvernementale de 7,13\% aux élections européennes de 2009 à 2,69\% en 2014. L'affirmationnisme blanc d'Aube dorée a ainsi pu se présenter comme un mode de régulation sociale et de palingénésie nationale. Dans la péninsule ibérique en crise, le populisme de droite demeure en revanche au point mort, alors même que Podemos et Ciudadanos représentent des critiques anti-establishment ayant renouvelé le logiciel idéologique de la gauche et du centre. La mémoire du franquisme rend quasiment impossible de mener une formation d'extrême droite dans les autonomies connaissant justement les plus fortes dynamiques nationalistes - le nationalisme catalan se vivant d'ailleurs comme une optimisation dans la globalisation, et non telle une enclosure. Au Portugal comme en Espagne, des tentatives de copie du FN ont été faites, en usant explicitement de sa flamme - que lui-même avait empruntée au Mouvement social italien (MSI) -, mais ces formations ne récoltent pas $1 \%$ des suffrages. 
En Allemagne, les expériences de constitution d'une offre national-populiste avaient toutes échoué de par le poids de la mémoire du nazisme. Le parti souverainiste Alternative pour l'Allemagne (AfD), fondé en 2013, s'est radicalisé en empruntant au néo-populisme, le menant certes à l'extrême droite mais ainsi défait de la problématique mémorielle. C'est la différence de mémoire qui, avec l'indifférenciation des offres politiques, a fourni l'énergie au Parti de la liberté d'Autriche (FPÖ). Fondé par d'ex-nazis en 1956, ayant mis sous le boisseau sa tendance national-allemande en 1993, il a pris un axe néolibéral qui, une fois aux affaires, a fait s'écrouler ses résultats électoraux à $10 \%$ en 2002. Dans l'opposition, il a repris un discours populiste qui lui a permis d'atteindre les portes de la présidence en 2016.

Jusqu'à l'éclosion électorale du FN en 1984, le MSI, fondé en 1946, était la formation nationaliste de référence. En 1969, l'ancien dignitaire fasciste GiorgioAlmirante en prit la tête. Recevant peu après F. Duprat, au nom du mouvement français Ordre nouveau $(\mathrm{ON})$, il lui déclarait la nécessité de « savoir pratiquer un fascisme souriant », formule que le Français réutilisera auprès des militants d'ON pour les convaincre de la stratégie de fondation du FN en $1972^{6}$. Le MSI aida au financement de ses homologues et organisa la liste de l'Eurodroite aux premières élections européennes (1979). Mais le «fascisme souriant » n'a pas suffi : le MSI n'a pu intégrer l'exercice du pouvoir qu'après son congrès de 1995, au cours duquel Gianfranco Fini proclama la mue dite « postfasciste ». Transformé en Alliance nationale, son réalignement vers un centre-droit gestionnaire l'a in fine électoralement tué. L'expérience Fini a été maintes fois citée par Marine Le Pen en contreexemple : intégrer culturellement le système revient à se désintégrer électoralement. Deux autres mouvements italiens ont récupéré le créneau.

Ce fut d'abord la Ligue du Nord pour l'indépendance de la Padanie, fondée en 1989. Sa participation au pouvoir l'a faite régresser aussi, mais son nouveau leader, Matteo Salvini, a laissé de côté la Padanie pour se concentrer sur une opposition tranchée à l'immigration, à l'islam, à l'Union européenne (UE) et aux réfugiés. Cette stratégie s'avère porteuse, la Ligue étant remontée de $4 \%$ des voix aux élections législatives de 2013 à $13 \%$ aux régionales de 2015. Elle est cependant désormais concurrencée par l'entrée en politique de Beppe Grillo. Fondé en 2009, son Mouvement 5 étoiles (M5S) a connu un succès fulgurant avec un programme social-populiste mais des élus très partagés dans leurs positions sur l'immigration ou le mariage des homosexuels. Ses eurodéputés ont rejoint le groupe fondé par le United Kingdom Independence Party (UKIP) au Parlement européen, mais ont tenté de le quitter pour rejoindre, sans succès, celui des eurolibéraux en janvier 2017.

Ce chaos participe de l'énergie du mouvement, mais il se comprend peut-être via l'exceptionnel score de populisme des sondés français se déclarant centristes : le positionnement « ni droite ni 
gauche » n'étant pas pour le M5S et pour ceux-ci la synthèse du centre eurolibéral, mais un bricolage transidéologique aboutissant parfois aux positions du centre - le Parti des Finlandais affirmant d'ailleurs un auto-positionnement centriste pour une politique alliant l'eurolibéralisme à la critique des élites et, surtout, de l'immigration.

Hormis la spécificité du cas grec, où les radicaux ont bénéficié de l'institutionnalisation des néopopulistes, les populistes des anciennes dictatures sont redevables du croisement de logiques de dégagement des mémoires et d'absence de débouchés pour la contestation sociale. Il semble que l'électorat récuse tant l'extrême droite néofasciste que la droite postfasciste, au bénéfice d'une offre anti-oligarchie et altérophobe.

\section{Populismes et démocraties illibérales}

Après le passage des anciennes démocraties populaires à la démocratie capitaliste, plusieurs formations à la fois populistes, ultranationalistes et xénophobes ont émergé, mais la situation coloniale qu'enduraient les pays du glacis soviétique explique aussi la présence d'un nationalisme transpartisan. Critiques envers les élites ayant émergé lors du passage à la démocratie de marché, exaltant une identité ethno-nationale, les formations populistes visaient, jusqu'à la récente crise des réfugiés, davantage les Roms, les juifs ou des minorités nationales que l'immigration. À l'Est, le vote en faveur de l'extrême droite semble recouvrir trois dimensions : un vote minoritaire de jeunes hommes, ouvriers ou chômeurs ; un vote de retraités paupérisés, parfois nostalgiques ; enfin, un vote paysan dont la cartographie s'oppose - à l'Est de la Pologne et de la Slovaquie, par exemple à un vote urbain davantage acquis aux formations libérales et social-démocrates. Extrêmes droites et droites radicalisées sont, selon les cas, en concurrence ou en complémentarité pour le positionnement populiste. Trois pays sont à cet égard remarquables : la Pologne et la Hongrie, initialement présentées comme des modèles de transition vers la démocratie de marché et incarnant aujourd'hui le modèle de la démocratie illibérale, et, cas spécifique, la Bulgarie.

Viktor Orbán, le leader de l'Union civique hongroise - affiliée au Parti populaire européen (PPE) qui regroupe tous les grands partis conservateurs de l'UE -, a reconquis le pouvoir en 2010 grâce à une campagne populiste, mais aussi grâce à la déstabilisation de rue que l'extrême droite radicale (Jobbik) a opposé au gouvernement sortant - les manifestants défilant avec le drapeau des Árpád, utilisé par les Croix fléchées, parti pro-nazi actif de 1939 à 1945, soulignant l'absence d'un processus social mémoriel dans les sociétés est-européennes. Pour autant, le gouvernement Orbán n'a nullement mis fin à l'approfondissement de la néolibéralisation de la Hongrie, mais a allié celleci à une pratique et un discours autoritaires de restriction des libertés fondamentales - ayant entraîné diverses protestations en Europe - et xénophobe - encore plus marqué depuis le début de la 
crise migratoire. Le durcissement continu du « régime Orbán » n’a pas non plus contenu le Jobbik, qui a obtenu 20,39\% aux élections législatives de 2014 : ainsi les radicaux permettent-ils aux illibéraux d'apparaître légalistes et conservateurs.

Si Viktor Orbán a indiqué, en 2014, que son pays devait se tourner vers les pays eurasiatiques non démocratiques mais performants dans la compétitionéconomique globalisée, plutôt que vers un Occident dont le libéralisme serait synonyme de déclin, le populisme polonais assume pour sa part une option pro-américaine dans sa critique de l'UE - comme en témoigne le déploiement sur son sol de troupes américaines en janvier 2017. La régression démocratique imposée par le gouvernement tenu par le parti Droit et justice (PiS) - affilié au groupe des Conservateurs et réformistes européens, au sein duquel siègent également les conservateurs britanniques - centralise les aspirations autoritaires, d'autant plus que l'offre partisane d'extrême droite est instable.

La Bulgarie, enfin, a connu une présence de l'extrême droite au second tour de son élection présidentielle de 2006, avec l'Union nationale Attaque fondée en 2005, celle-ci critiquant l'UE, les

élites atlantistes, les Roms et la minorité turque. À la fois anti-israélien et islamophobe, le parti a su mobiliser l'électorat miné par la marche vers l'Ouest et par la mémoire de l'Empire ottoman - le parti représentant la minorité turque ayant un rôle de parti-pivot, le rejet de cette minorité est tant celle de l'Orient que de l'Occident. Le soutien sans participation à la majorité gouvernementale de 2013 a depuis fait s'écrouler les scores de la formation.

La diversité des cas témoigne néanmoins d'une logique : ces dynamiques ne sont plus seulement attribuables à la situation postcommuniste de ces pays, mais relèvent aussi des effets de l'élargissement de l'UE et des déstabilisations induites, ainsi que de l'onde de choc de l'enchevêtrement des crises économiques et géopolitiques de ces dernières années - soit la même mécanique qui a bouleversé le paysage droitier transatlantique.

\section{Conclusion}

Crise économique, « printemps arabes », terrorisme, crise des réfugiés : l'autoritarisme unitariste s'appuie sur le désarroi identitaire, comme en attestent les polémiques sur les modes de vie amenant aux interdictions des abattages halal et casher en Pologne - en 2013, levées en 2014 - ou au Danemark (2015). La critique du multiculturalisme aboutit à une remise en cause du libéralisme culturel à l'Ouest et politique à l'Est. Elle épargne, en revanche, le libéralisme économique : la participation des populistes à des coalitions se fait sur des programmes eurolibéraux, complétés par la lutte contre l'immigration.

Les partis populistes permettent ainsi de construire un bloc politique d'alliance entre des catégories sociales ayant des intérêts économiques divergents, assurant la tenue de politiques eurolibérales, en 
les inclinant en un sens pour eux essentiel : la lutte contre l'immigration et le combat identitaire. Ils participent d'un libéralisme ethnicisé sous les contrecoups de la conjonction des crises. Le contrecoup pour eux est que cela les rend souvent fragiles lors de l'exercice des responsabilités, mais ils bénéficient d'une mansuétude de leurs électeurs.

L'extension du populisme dans l'espace libéral, ainsi au centre, correspond à un autre type de réaction à ces crises, avec la volonté de contenir la dénonciation du caractère postdémocratique de l'UE, ou, dans le cas de la droite américaine, de réinstaurer une verticalité face à l'extension des droits des individus et au multiculturalisme - le vote Trump ayant rassemblé les électeurs aux revenus supérieurs à la médiane, tandis que le vote Sarkozy s'expliquait par le thème d'une valeur travail censée restaurer une hiérarchie sociale légitime, alors que François Fillon a joué l'ordolibéralisme à l'assaut des contre-pouvoirs.

La question politique est celle du rapprochement possible entre droitisation transatlantique et démocraties illibérales orientales. Depuis le premier choc pétrolier, l'espace transatlantique connaît une droitisation qui recouvre un démantèlement de l'État social et de l'humanisme égalitaire au profit d'un accroissement de l'État pénal et d'une ethnicisation des questions et représentations sociales. Ce processus porte une demande sociale autoritaire qui est une réaction à la transformation et à l'atomisation des modes de vie et de représentations dans un univers économique globalisé, financiarisé, dont l'Occident n'est plus le centre.

À l'Est du continent européen, les démocraties illibérales qui se constituent viennent donner une forme institutionnelle à cette droitisation transatlantique, et par là même présentent un débouché politique crédible pour les populistes, en particulier pour les extrêmes droites qui ne présentent plus un projet d'instauration de régimes autoritaires, mais s'affichent comme une offre de gouvernance autoritaire dans le cadre démocratique. Le passage d'une offre de régimes à celle de partis se constituant tels des lobbies ethnoculturels au sein d'un ordre institutionnel pluraliste démontre l'adaptabilité d'une vision du monde née au XIXe siècle au contexte géopolitique fluctuant actuel. Mais la rencontre éventuelle entre ces dynamiques n'a rien d'automatique. La demande autoritaire et unitariste peut trouver une issue hors de l'autoritarisme. En France, lorsque la vague poujadiste disparaît en 1958 aussi soudainement qu'elle était apparue, ce n'est pas qu'elle fut un « feu de paille », selon une analyse classique. Les questions fiscale et algérienne qui la motivaient n'avaient pas non plus disparu. Mais l'arrivée au pouvoir du général de Gaulle et le remplacement de la IVe République par la Ve avaient satisfait le désir d'ordre et d'autorité au bénéfice de la perpétuation de la démocratie libérale.

\section{Notes}


François Duprat, « Le Néo-fascisme en Occident. II : Amérique latine », Revue d'Histoire du fascisme, $\mathrm{n}^{\circ} 13$, supplément, novembre 1975, p. 25.

Gaël Brustier et Jean-Philippe Huelin, Voyage au bout de la droite, Paris, Mille et une nuits, 2011. Ifop, «L'attirance des Français pour un gouvernement technocratique ou autoritaire », 2 novembre 2015 ; Ipsos-Steria, « Fractures françaises », 6 mai 2015.

Gilles Ivaldi, Andrej Zaslove et Agnes Akkerman, « La France populiste ? », Note de recherche CEVIPOF, $\mathrm{n}^{\circ} 30$, vague 7, février 2017.

Voir Céline Braconnier et Nonna Mayer, Les Inaudibles. Sociologie politique des précaires, Paris, Presses de Sciences Po, 2015.

Voir Direction des renseignements généraux, « Activités à l'étranger d'Ordre Nouveau », 9 septembre 1971, p. 3, AN/20080389/47; « Ordre Nouveau et les élections législatives », Bulletin hebdomadaire, 31 décembre 1971, p. 1, AN/F/7/15574. 